\title{
Acción docente en situaciones de aprendizaje conceptual
}

\section{Teaching action in situations of conceptual learning}

\author{
Ramón Martínez, \\ Xiomara Arrieta, \\ Víctor Riveros \\ Doctorado en Ciencias Humanas, \\ Facultad de Humanidades y Educación, \\ Universidad del Zulia
}

\section{Resumen}

Los estudiantes de ciencias experimentales evidencian una escasez de conocimientos científicos previos a las actividades de las clases teóricas, resolución de problemas y de prácticas de laboratorio, que revelan aprendizajes mecánicos de los procesos aplicados, sin argumentaciones fundamentadas que den respuesta a situaciones problemáticas que se les presentan. Ante esta situación, la acción docente para enfrentar esta problemática es un reto impostergable. El objetivo de esta investigación es diseñar una estrategia de acción docente que promueva el aprendizaje significativo de conceptos científicos en el contexto del aula universitaria. La metodología es documental descriptiva. La estrategia, apoyada en la teoría de los campos conceptuales, integra éste con el aprendizaje como investigación científica dirigida, el estudio de la interacción en el aula y el aprendizaje significativo, conducente a una enseñanza eficaz de la conceptualización de lo real científico. 
Palabras clave: Campos conceptuales, aprendizaje significativo, enseñanza eficaz, aprendizaj e como investigación científica dirigida, acción docente.

Abstrac: Experimental Science students show a lack of scientific knowledge before theoretical class, problem solving and laboratory practices that reveal mechanical learning process applied without substantiated arguments that respond to problematic situations the face. In this situation, the teaching action to address this problem is an urgent challenge. The objective of this research is to design a strategy that promotes teaching activities meaningful learning of scientific concepts in the context of the university classroom. The methodology is descriptive documentary. The strategy, based on the theory of conceptual fields, integrates this with learning as directed scientific research, the study of classroom interaction and meaningful learning, conducive to effective teaching of conceptualization of scientific reality.

Key words: conceptual fields, significant learning, effective teaching, learning and scientific research directed, teaching action.

\section{Introducción}

Los estudiantes de las asignaturas de las ciencias experimentales, sobre todo en los dos primeros semestres de las carreras universitarias, evidencian una escasez de conocimientos científicos previo a las actividades de las clase teóricas, resolución de problemas y actividades de laboratorio, que revelan la mecanización y matematización de los procesos aplicados, sin argumentos ni razonamientos que den respuesta a las situaciones problemáticas planteadas. La acción docente ante este déficit cognitivo de los estudiantes es un reto a enfrentar.

Por tal motivo, la investigación, documental descriptiva, se orienta a la búsqueda de una estrategia de acción docente que mejore el proceso de enseñanza enfocado en el aprendizaje significativo de conceptos en el contexto del aula universitaria, y considere las diferencias cognoscitivas individuales en su proceso de aprendizaje. 
Se formula la siguiente pregunta de investigación: ¿es posible una estrategia de acción docente que posibilite el aprendizaj e significativo de la conceptualización científica en las asignaturas de las ciencias experimentales, a partir de la integración de diversas teorías consideradas, conducente a una enseñanza eficaz centrada en el alumno, en el contexto del aula universitaria?

El objetivo de la presente investigación es diseñar una estrategia de acción docente que promueva el aprendizaje significativo de conceptos científicos en el contexto del aula universitaria. La estrategia para alcanzar este objetivo establece los siguientes aspectos a) relación entre la teoría de los campos conceptuales con la teoría de aprendizaj e verbal significativo; b) descripción de las bases estratégicas del aprendizaje significativo y su conexión con los estilos de aprendizaj e; c) definición de enseñanza eficaz a partir del estudio de las prácticas educativas y de la interacción en el aula; d) construcción de los elementos de una estrategia de acción docente que promueva el aprendizaje significativo de conceptos científicos en el contexto del aula universitaria.

Pero, ¿por qué conceptos? El hombre vive en un mundo de conceptos en lugar de objetos, acontecimientos y situaciones. Los hechos reales del mundo físico se perciben a través de categorías, en sus versiones simplificada, abstracta y generalizada. Sin ellos no hay comprensión, no hay desarrollo cognoscitivo. Consecuentemente, los conceptos deberían ser el centro de las actividades de enseñanza y aprendizaje, por lo que se debe dar el importante papel de los conceptos en la educación científica (Moreira, 2010).

\section{Integración de la teoría de los campos conceptuales y la teoría del aprendizaje verbal significativo. Implicaciones en la acción docente}

Ambas teorías psicológico-cognitivas presentan aspectos que los diferencian y asemejan. Sin considerar los aspectos que las distinguen, un aspecto en común de ambas teorías se refiere al enfoque dado a los conceptos, con implicaciones pedagógicas.

El campo conceptual es a la vez el conjunto de las situaciones, de 
Ramón Martínez, Xiomara Arrieta, Víctor Riveros

conceptos y proposiciones que permiten analizarlas como tareas. Estos conceptos no van solos, tendrían alcance limitado si las proposiciones científicas no se les da su función en el tratamiento de las situaciones. En efecto, los campos conceptuales identifican dos entradas: la primera, son las situaciones, y la segunda, los conceptos y proposiciones.

No es una teoría de enseñanza de conceptos explícitos y formalizados. Es una teoría psicológica de la conceptualización de lo real que permite identificar y analizar las filiaciones y rupturas entre conocimientos desde el punto de vista de su contenido conceptual (Vergnaud, 1990). En el estudio de este proceso, la conceptualización de lo real permite el análisis conceptual de dominio de contenidos específicos del conocimiento. El interés por lo que sucede en el aula determina la importancia por este contenido y el estudio del funcionamiento cognitivo del sujeto-en-acción.

Una ventaja de la aproximación de campos conceptuales es la de permitir generar una clasificación de las situaciones que reposa sobre el análisis de las tareas cognitivas y en los procedimientos que pueden ser puestos en juego en cada una de ellas. Para ello es importante conocer la naturaleza y la dificultad propias de cada una de las tareas y subtareas.

Si se está interesado en el aprendizaj e y la enseñanza de un concepto, éste no puede reducirse a su definición. Es esencial para la didáctica un proceso de elaboración utilitarista de situaciones variadas y de problemas que se pretenden resolver para que el concepto tenga sentido para el aprendiz. El núcleo de esta elaboración está en el modo que toma la acción del sujeto si se quiere considerar correctamente la magnitud de la función adaptativa del conocimiento racional. El conocimiento racional es operatorio o no es tal conocimiento (Vergnaud, 1990).

Este enfoque requiere para su retención en la memoria semántica que el aprendizaje sea significativo, y la significatividad en la operacionalización de los conceptos ante campos conceptuales organizados, solo es posible, en el marco de las relaciones del aprendizaje significativo, significatividad potencial, significatividad 
lógica y significado psicológico, y de sus condiciones, criterios y variables. De este modo, la teoría del aprendizaje verbal significativo proporciona a la acción docente los principios pedagógicos para la enseñanza en el aula.

Ahora, ¿cómo se integra la teoría ausubeliana en la teoría de los campos conceptuales hacia la búsqueda de los elementos de diseño? La palabra clave de esta integración es el significado. El significado del concepto vergnaudiano por ser implícito, es psicológico. El significado psicológico ausubeliano es idéntico al real o fenomenológico, el cual es producto de un proceso de aprendizaje significativo. Surge cuando el significado potencial se convierte en un contenido cognoscitivo nuevo, diferenciado e idiosincrático, como resultado de haber sido relacionado de modo no arbitrario y sustancial con las ideas relevantes de su estructura cognoscitiva, así como también de haber interactuado con éstas (Ausubel, Novak y Hanesian, 2009).

El significado potencial depende de la significación lógica y la disponibilidad de las ideas pertinentes en la estructura cognoscitiva del alumno individual, en la que se pueden involucrar los invariantes operatorios de los esquemas, por lo que existe coincidencia entre los significados vergnaudianos y ausubelianos, lo cual permite su integración en los elementos del diseño.

La visión conjunta de ambas teorías posibilita entonces el reforzamiento de la efectividad del alcance del aprendizaje significativo de conceptos, durante la operacionalización de los mismos, en campos conceptuales intencionados, lo que abre múltiples posibilidades para la docencia.

Partiendo de estos aspectos, el aprendizaje verbal significativo prevalecerá en la generación de una estrategia de acción docente en la enseñanza de conceptos y proposiciones explícitas en el aula universitaria.

En este sentido, Vergnaud (1990) define el concepto como un conjunto de invariantes operatorios utilizables en la acción, en la que se ponen en juego el conjunto de situaciones que constituyen la 
referencia de sus diferentes propiedades, y el conjunto de los esquemas evocados por los suj etos en estas situaciones. En efecto, cada uno de los conceptos comporta varias propiedades, cuya pertinencia es variable según las situaciones a tratar. Específicamente, es la consideración simultánea de una tripleta de conj untos que permiten el estudio del desarrollo y el funcionamiento cognoscitivos del concepto, ya sea en el curso del aprendizaje o durante su uso. Estos son:

a) El referente: conjunto de las situaciones que dan sentido al concepto.

b) El significado: conjunto de los invariantes operatorios sobre los cuales reposa la operacionalidad de los esquemas.

c) El significante: conjunto de las formas lingüísticas y no lingüísticas que permiten representar simbólicamente el concepto, sus propiedades, las situaciones y los procedimientos de tratamiento.

La importancia del aprendizaje significativo en la adquisición del conocimiento radica en el conocimiento pertinente existente en la estructura cognoscitiva del sujeto, el cual permite facilitarlo. Para la teoría de los campos conceptuales, el acercamiento entre lo real y la representación debe ser buscado en primer lugar al nivel de los invariantes operatorios contenidos en los esquemas. Para ambas teorías los conocimientos previos de los alumnos son la base de la conceptualización de lo real.

Al respecto, los invariantes operatorios se pueden considerar como el constructo clave de los conocimientos previos contenidos en un esquema. Estos invariantes ocupan la mayor parte de la conceptualización de lo real, pero no son independientes de los conceptos; sin ellos no existirían los conceptos y proposiciones explícitos de la ciencia; progresivamente se van modificando hasta que se evidencia la automatización de los esquemas de asimilación ante una clase dada de situaciones.

Estas ideas son fundamentales para la comprensión del modelo de aprendizaje ausubeliano que, en esencia, relaciona las ideas expresadas simbólicamente con lo que el alumno ya sabe, de modo no arbitrario y sustancial (no al pie de la letra). Estas características, 
esenciales para un aprendizaj e significativo de conceptos, relacionan las ideas nuevas con conceptos específicamente relevantes existentes en la estructura cognoscitiva del alumno en particular. Para que el concepto tenga sentido para él, de acuerdo a la postura vergnaudiana, los esquemas evocados se deben relacionar a las situaciones y a los significantes. De allí se infiere que las criterios de no arbitrariedad y sustancialidad deben considerarse en las situaciones y los significantes.

En la contemplación de estos aspectos en la enseñanza de las ciencias, la mediación es un proceso importante. Es necesario analizar en qué consisten los actos de mediación del profesor en las situaciones de enseñanza, en los cuales el lenguaje juego un papel fundamental

El primer acto de mediación de la enseñanza es la elección de la situación o las clases de situaciones a proponer a los alumnos. En ella, el profesor puede encontrar oportuno identificar e introducir las filiaciones y las rupturas. En un segundo acto de mediación, los profesores acompañan por el lenguaje a las actividades de los alumnos en su desarrollo cognoscitivo, y por tanto, al proceso de elaboración de los ingredientes de los esquemas: metas y anticipaciones, reglas de acción, invariantes operatorios y posibilidades de inferencia (Vergnaud, 2007). Para el autor, no tiene sentido el aprendizaje si no se desestabiliza a los alumnos, aunque demasiada desestabilización interfiere en el aprendizaj e. En este proceso el trabajo de mediación del profesor es más complejo pero más importante.

Como elemento estratégico del primer acto de mediación se introduce el uso de materiales introductorios, como los organizadores previos, que permiten desarrollar los invariantes operatorios antes de la enseñanza formal. Este desarrollo se puede lograr con fines educativos de dos maneras: sustancialmente y programáticamente. Sustancialmente, empleando, con propósitos de organización e integración, aquellos conceptos y proposiciones unificadores de una disciplina dada que tengan dominios más explicativos, de inclusividad, generalidad y relacionabilidad con el contenido programático de la asignatura en cuestión; programáticamente, para organizar la secuencia de la materia de estudio, construir su lógica y organización internas y preparar ensayos de prácticas. Son aplicables dos principios 
que enfatizan en el aprendizaje de conceptos: la diferenciación progresiva y la reconciliación integradora.

Asimismo, el primer acto de mediación tiene implicaciones en la acción docente, producto de la integración de las teorías de los campos conceptuales y el aprendizaje verbal significativo, la cual no sería posible si no se cumplen las condiciones para que el aprendizaje de la conceptualización científica sea significativo para el alumno. La primera condición es la disposición de aprender significativamente, en caso contrario el alumno aprenderá de manera repetitiva, mecánica, carente de significado. La segunda condición es que los materiales de estudio sean potencialmente significativos, es decir, que tengan significación lógica y que puedan vincularse con los invariantes operatorios disponibles en los esquemas de los aprendices.

Por otro lado, en el segundo acto de mediación se aprecia la importancia atribuida a la interacción social, al lenguaje y a la simbolización en el dominio progresivo de un campo conceptual.

La interacción social involucra las diadas al umnos-alumnos y alumnosprofesor. La interacción de las diadas mencionadas con los materiales de estudio potencialmente significativos es un compartir de significados entre alumnos y el profesor con respecto a los conocimientos guiados por los materiales educativos del currículo. En este sentido, El aporte de Gowin (1981) a la teoría del aprendizaje verbal significativo establece una relación triádica alumno-profesormateriales educativa que tiene por propósito la asimilación de la conceptualización científica, aceptada por la comunidad científica, encabezada por el profesor y el material de instrucción potencialmente significativo.

Para la materialización de la interacción social, que lleve a la captación de significados y al aprendizaje significativo, se propone las actividades colaborativas en la realización conjunta de tareas de aprendizaje en pequeños grupos, en las que el lenguaje implica un mínimo de dos personas intercambiando significados de manera recíproca (Moreira, 2010), comprometidos responsablemente en el logro de las metas grupales. Esta interacción es dinámica ya que en 
ella se comparten y negocian significados entre alumno y profesor y entre alumnos, en el cual es fundamental la función de comunicación del lenguaje. La actividad lingüística ayuda a generar los esquemas en función de las características particulares de cada una de las situaciones de la clase hasta que éstas sean lo suficientemente dominadas. En ese proceso el aprendiz se involucra en la tarea, facilitándole la expresión y la simbolización de los conceptos, y permitiéndole de esta forma emitir juicios que logren externalizar los significados que está captando. Se infiere entonces que la interacción social y el lenguaje son condiciones necesarias para el aprendizaje significativo.

Con relación al papel del docente en la interacción social, su función principal, aparte de enseñar, debe ser la de mediador de los aprendizajes, complementada con otras funciones como amigo, consejero, orientador, representante de la cultura, transmisor de los valores, facilitador del desarrollo de la personalidad.

Como estrategia facilitadora del aprendizaj e significativo, se propone a los mapas conceptuales (Moreira, 2010), por ser mapas de conceptos y proposiciones, para apreciar de manera representativa la transferibilidad de las funciones proposicionales a argumentos, decisivos en la conceptualización de lo real científico. Por ser instrumentos de negociación de significados y externalización de significados psicológicos, deben ser construidos y reconstruidos por los alumnos en actividades colaborativas, ya que estos lo promueven naturalmente. En este proceso los alumnos discutirán los conceptos y proposiciones a incluir en el mapa, como serán jerarquizados, cuáles serán las relaciones a priorizar y cuales los conectivos a usar para explicitar tales relaciones, y en este proceso lo van exponiendo a sus compañeros y profesores.

\section{Bases estratégicas de enseñanza}

De la integración de las teorías señaladas previamente surgen dos bases estratégicas de enseñanza para el aprendizaje significativo de la conceptualización científica: los organizadores previos y los mapas conceptuales. Sin embargo, ambas bases no son las únicas: objetivos o intenciones, actividad focal introductoria, discusión 
guiada, actividad generadora de información previa, resumen en clase, analogías, señalizaciones orales. Los criterios para su elección dependerá básicamente del contenido del conocimiento enfocado: el aprendizaje de conceptos y de proposiciones científicas, así como también a la modalidad de la enseñanza: verbal expositiva, diseño del material potencialmente significativo, semipresencial o aula virtual, es decir, actividades de la modalidad declarativa. Para las actividades de la modalidad procedimental, como los trabajos de laboratorio, una base estratégica muy usada es la $V$ epistemológica.

Estas bases pueden emplearse antes, durante y al término de la situación de enseñanza, e incluso, como en el caso de los mapas conceptuales, en cualquier momento de la enseñanza, y deben ser procedimientos utilizados en forma reflexiva y flexible por el docente. Las bases estratégicas utilizadas antes de la situación de enseñanza o preinstruccionales pueden emplearse para la activación de los conocimientos previos o para tender puentes entre estos últimos y el nuevo. Las utilizadas durante la situación de enseñanza o coinstruccionales pueden favorecer la atención, la codificación y/ o el procesamiento profundo de la información. Otras estrategias son más útiles al término de la situación de enseñanza o postinstruccionales para reforzar el aprendizaje de la información nueva.

\section{Estilos de aprendizaje individual}

Los estudiantes del nivel universitario, aprenden de manera diferente en el aula; el papel del docente es determinar cómo aprenden sus estudiantes para diseñar clases de situaciones y estrategias didácticas que le faciliten la construcción de aprendizaj es significativos cónsonos con el conocimiento científico. La efectividad en el aprendizaje depende en gran medida del aj uste de los estilos de aprendizaje de los alumnos a los estilos de enseñanza (o estilos de aprendizaje a nivel profesoral), por eso es de vital importancia que el docente conozca los diferentes estilos de cómo sus alumnos aprenden significativamente.

Existe una gran cantidad y diversidad de definiciones de estilos de aprendizaje; sin embargo, Keefe y Ferrell (1990) la aceptan como 
el conjunto de rasgos cognoscitivos, afectivos y fisiológicos, que sirven como indicadores relativamente estables, de cómo el aprendiz percibe, interactúa con su entorno y responde a él. Esta definición posee una visión holística, basada en la idea de que los mismos son producidos por la interacción de factores genéticos, biológicos y ambientales.

La figura 1 representa uno de los modelos de estilos de aprendizaje más aceptados: el de Aprendizaje Experiencial de Kolb (1984). Para que el aprendizaje sea efectivo requiere de cuatro clases de habilidades: Experiencia Concreta (EC), Observación Reflexiva (OR), Conceptualización Abstracta (CA) y Experimentación Activa (EA).

La experiencia concreta inmediata (EC) es la base de la reflexión y la observación. Un uso individual de estas observaciones permite construir un concepto, proposición, o "teoría" de la cual se pueden deducir nuevas implicaciones para la acción. En ella, el aprendiz debe ser capaz de involucrarse total, abierta y sin parcialidad en experiencias nuevas. Debe ser capaz de reflejar y observar sus experiencias desde muchas perspectivas (OR). Debe ser capaz de crear conceptos que integren sus observaciones en teorías que suenen lógicas (CA), y debe ser capaz de usar estas teorías para tomar decisiones y resolver problemas (EA). El aprendizaj e se concibe como un ciclo de cuatro estados (figura 1).

La categoría de la cognición (percibir, averiguar, obtener información) es caracterizado en lo abstracto/concreto; la categoría de la conceptualización (pensamiento, formación de ideas, procesamiento, memoria), en la observación reflexiva/ experimentación activa (figura 1).

Para Kolb (1984), las dos dimensiones de abstracto-concreto y activo-reflexivo identifican cuatro estilos de aprendizaje: el convergente, el divergente, el asimilador, y el acomodador (Tabla 1). Estos estilos relacionan la captación (percepción) de la experiencia con su transformación (procesamiento) a través, respectivamente, de la dialéctica abstracto/ concreto, representada por los procesos de comprensión y aprehensión, y la dialéctica activo/ reflexivo, representada por los procesos de intensión y extensión. 
Los procesos de comprensión y aprehensión, asociados a la percepción del conocimiento, y los procesos de intensión y extensión, asociados al procesamiento de la información, se definen (Salas, 2008).

a) Comprensión: El aprendiz confía en la interpretación y representación simbólica en su captación de la experiencia del mundo (Abstracto).

b) Aprehensión: El aprendiz confía en las cualidades tangibles y sentidas de la experiencia inmediata (Concreto).

c) Intención: El aprendiz procesa la captación de la información a través de una reflexión interna (Reflexivo).

d) Extensión: El aprendiz procesa la captación de la información a través de una manipulación activa del mundo externo (Activo).

Tabla 1

Estilos de aprendizaje individual

\begin{tabular}{|c|c|c|}
\hline Estilo de Aprendizaje & $\begin{array}{c}\text { Captación y } \\
\text { transformación }\end{array}$ & Habilidad \\
\hline Divergente & $\begin{array}{l}\text { La experiencia es } \\
\text { percibida por medio de } \\
\text { la aprehensión, y } \\
\text { procesada a través de } \\
\text { la intensión. }\end{array}$ & $\begin{array}{l}\text { Su mayor fuerza reside } \\
\text { en la capacidad } \\
\text { imaginativa. }\end{array}$ \\
\hline Asimilador & $\begin{array}{l}\text { La experiencia es } \\
\text { percibida por medio de } \\
\text { la comprensión, y } \\
\text { procesada a través de } \\
\text { la intención. }\end{array}$ & $\begin{array}{l}\text { Su mayor fuerza reside } \\
\text { en la capacidad para } \\
\text { crear modelos teóricos }\end{array}$ \\
\hline Convergente & $\begin{array}{l}\text { La experiencia es } \\
\text { percibida por medio de } \\
\text { la comprensión, y } \\
\text { procesada a través de } \\
\text { la extensión. }\end{array}$ & $\begin{array}{l}\text { Su mayor fuerza reside } \\
\text { en la aplicación práctica } \\
\text { de las ideas. }\end{array}$ \\
\hline Acomodador & $\begin{array}{l}\text { La experiencia es } \\
\text { percibida por medio de } \\
\text { la aprehensión, y } \\
\text { procesada a través de } \\
\text { la extensión. }\end{array}$ & $\begin{array}{l}\text { Su mayor fuerza radica } \\
\text { en hacer las cosas, en } \\
\text { la realización de planes } \\
\text { y experimentos y } \\
\text { participar en nuevas } \\
\text { experiencias. }\end{array}$ \\
\hline
\end{tabular}

Fuente: Kolb,(1984).

Adaptado por Martínez, Arrieta y Riveros (2013) 
Figura 1

Rueda de Kolb, modificado

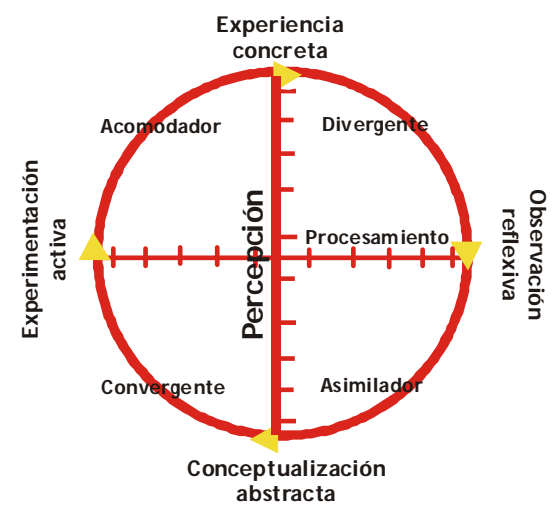

Fuente: Martínez, Arrieta y Riveros (2013)

Es importante destacar que estos estilos de aprendizaje no deben convertirse en estereotipos. Hay estilos más sobresalientes que otros, hasta alcanzar el más predominante.

\section{Enseñanza eficaz}

Aunque es necesaria la integración de las teorías ausubeliana, vergnaudiana y kolbiana para que la enseñanza sea eficaz en lograr resultados observables de aprendizaje significativo de la conceptualización científica, centrado en el alumno, ésta se hace suficiente si la acción docente adapta esta integración a la realidad de su contexto, solo posible si se observa e interpreta la interacción en el aula, consciente, a su vez, de las transformaciones necesarias de las situaciones, que conlleven a su integración y permitan conducir el aprendizaj e como de investigación científica dirigida.

Al respecto, se intenta dar una definición de enseñanza eficaz a partir de la integración de los elementos académicos involucrados en el hecho educativo: aprendizaje de conceptos, resolución de problemas de lápiz y papel, prácticas de laboratorio y evaluación (Gil et al., 1999), por una parte, y por la otra, la descripción de la práctica educativa y la interacción en el aula a partir de los estudios recientes sobre el quehacer en el aula. 
La integración se inicia con la necesidad de la transformación efectiva de la enseñanza tradicional (que concibe a los estudiantes como simples receptores) de las ciencias a la introducción de innovaciones en su didáctica, permitiendo la construcción de un cuerpo de conocimientos en el que se integren coherentemente los elementos académicos, que conciba a los estudiantes como investigadores noveles, integrados en equipos cooperativos, a su vez inmersos en el proceso de negociación de significados entre ellos y el resto de la comunidad científica encabezada por el profesor y el material potencialmente significativo.

Con relación al aprendizaje como investigación científica dirigida, esta se fortalece con los aportes de la Teoría de los Campos Conceptuales de Vergnaud (1990). Ello permite la creación de un Modelo Dinámico de Aprendizaj e en los Trabajos de Iaboratorio en Física (Andrés, Pesa y Moreira, 2006), en la que cada trabajo experimental debe tener explícitos obj etivos de aprendizaje asociados con lo conceptual y lo epistemológico, conducentes a la integración de lo conceptual con lo real, conectar dialécticamente los datos y la teoría y, sobre todo, la promoción del desarrollo de una visión de la naturaleza de la ciencia cercana al quehacer científico.

Por otra parte, para la teoría vergnaudiana, la resolución progresiva de situaciones problemáticas potencia a los alumnos a mostrar mayor dominio de los conceptos y proposiciones explícitas, quedando habilitados para transferirlos a nuevos problemas de mayor complejidad (Meleán y Arrieta, 2009). La consecuente automatización en la organización de la conducta ante clases de situaciones similares conduce a una conceptualización implícita más elaborada y estable, y por consiguiente significativa. La resolución de problemas es un método válido y práctico para evidenciar el aprendizaj e significativo de las ideas verbalizadas.

Vergnaud (1990) establece también un nuevo estatus a las situaciones de ruptura cognoscitiva, ya que suponen la sustitución del cuestionamiento externo de los invariantes operatorios de los estudiantes por la de un diálogo abierto que oriente la resolución de problemas abiertos por ellos. 
La integración de las prácticas de laboratorio y la resolución de problemas las ubica en el tratamiento de situaciones problemáticas abiertas, con una orientación aproximada de la actividad científica. La puesta a prueba de una hipótesis, en una investigación científica, se valida experimentalmente y se implica coherentemente con el cuerpo de conocimientos aceptado por la comunidad científica.

A su vez, la evaluación es parte integral de una buena enseñanza. Le aporta al docente la regulación y el conocimiento de los factores y problemas que promueven el proceso de enseñanza y aprendizaje. Sin esta actividad sería difícil asegurarle al profesor el logro del aprendizaje de los alumnos; le costaría saber sobre los resultados y la eficacia de la acción docente y de los procedimientos de enseñanza usados. Tampoco tendría argumentos suficientes para proponer correcciones y mejoras (Díaz-Barriga y Hernández, 2010). Para estos autores, la evaluación constructivista de los aprendizajes, ya sea diagnóstica, formativa o sumativa, presenta dos funciones: la pedagógica y la social.

La función pedagógica de la evaluación de los aprendizajes permite obtener información sobre cómo va evolucionando el aprendizaje durante el proceso, así como lo sucedido con las estrategias de enseñanza, para proporcionar ayudas en el momento en que se detecten los problemas, plantear actividades de refuerzo o ampliación según el grado de consecución de los objetivos o competencias y reorientar la planificación de las secuencias de aprendizaje. La función social es acreditativa. Da cuenta del grado de logro de los obj etivos o competencias propuestos. Se da al final de cada ciclo de la asignatura y al final de ésta. La acreditación obtenida se basa en la calificación, la promoción o no, la titulación o no y la orientación al final del proceso.

Por otro lado, según Coll y Sánchez (2008), la crisis del modelo que establece una relación epistemológica unidireccional y jerárquica entre la investigación académica, y la práctica profesional y la importancia creciente otorgada al contexto del aula en el estudio de la interacción educativa, están en el origen del auge actual del estudio de las prácticas educativas y de la interacción en el aula. Estos cambios se han venido produciendo en el transcurso de las 
Ramón Martínez, Xiomara Arrieta, Víctor Riveros

últimas dos o tres décadas.

La crisis del modelo citado conlleva a una relación bidireccional y de interdependencia entre investigación y profesión. De los factores de este cambio destaca las dificultades del profesorado por integrar a su acción docente las orientaciones formuladas por las investigaciones pedagógicas, así como las dificultades asociadas a los intentos de uso de las aportaciones de la investigación educativa. De allí la importancia de una relación dialógica entre investigación y acción docente.

Pero, ¿qué se investiga? La organización del conjunto de situaciones a partir de ideas semejantes hace fecunda la investigación de las filiaciones y rupturas entre conocimientos (saber-hacer y saberes expresados), para comprender en qué consiste tal o cual concepto, desde el punto de vista cognitivo (Vergnaud, 1990). Esta investigación constituye la finalidad principal de la teoría de los campos conceptuales y forma parte de la investigación de la interacción social en el aula, ya que el significado del concepto se comparte y permite la comunicación. A partir de estos estudios se concibe a la práctica educativa como una fuente inapreciable de conocimientos que intenta comprender cómo se produce la interacción entre los procesos de enseñanza y aprendizaje.

El segundo cambio parte de los planteamientos de que la construcción del conocimiento es inseparable del contexto o contextos en el que se adquiere y utiliza. En ese sentido comienza a diferenciarse entre contexto físico y contexto mental. El primero representa las características espaciales, materiales, entre otros; el segundo, a las representaciones, expectativas, afectos, motivaciones, entre otros. Pero el cambio más importante se produce en el traspaso de esta diferenciación y su incidencia sobre el proceso de enseñanza y aprendizaj e a una visión del aula como un contexto de enseñanza y aprendizaje, construido por docentes y estudiantes, mediante las actividades que en ella se llevan a cabo.

Es así, que en los procesos de enseñanza y aprendizaje, como construcción conjunta de significados, los cambios observables en los alumnos se relacionan con los recursos discursivos del profesorado. 
En aulas en las que los profesores utilizan una estructura de actividad, los estudiantes aprenden a expresar sus puntos de vista de los contenidos con su propio discurso a medida que las tareas adquieren significado en el contexto de la clase. La negociación de significados de los profesores y alumnos en los procesos de enseñanza y aprendizaje, en la que entran en juego una serie de medios discursivos que favorecen el diálogo, producen cambios en la comprensión de éstos durante las actividades de aula (Cubero et al., 2008).

En consecuencia, la enseñanza eficaz es una conceptualización dinámica que depende del estudio y divulgación, por parte del profesorado, de la práctica educativa y su interacción discursiva con los alumnos, de ellos entre sí, con los materiales de estudio potencialmente significativos, como una forma de negociación de significados conducentes a la significatividad psicológica de los saberes, por parte de los alumnos, ante variedades situacionales que integran el aprendizaje teórico, la resolución de problemas, las experiencias de laboratorio y la evaluación constructivista en el contexto construido por estos actores del hecho educativo, hacia la conceptualización observable de la realidad científica representada y explicada por los estudiantes en su propio discurso.

Para efecto de la presente investigación, la acción docente es la actividad de aplicación adecuada de estrategias de enseñanza de los factores cognoscitivos en las situaciones de aprendizaje significativo conceptual (estructura cognitiva y transferencia, desarrollo cognitivo y disposición, capacidad intelectual, diferencias individuales en la capacidad intelectual), así como las estrategias de apoyo que contemplan los factores afectivos, holísticos y sociales del aprendizaje (motivacionales, de la personalidad, grupales y sociales), y las estrategias facilitadoras del aprendizaje (mapas conceptuales, la V de Gowin, la tarea docente desde el punto de vista constructivista).

\section{Elementos de una estrategia de acción docente}

Los elementos que constituyen las bases para el diseño y generación de una estrategia de acción docente, que promueva el aprendizaje significativo de conceptos y proposiciones científicos en el contexto del aula universitaria, se describen en la tabla 2. 
Ramón Martínez, Xiomara Arrieta, Víctor Riveros

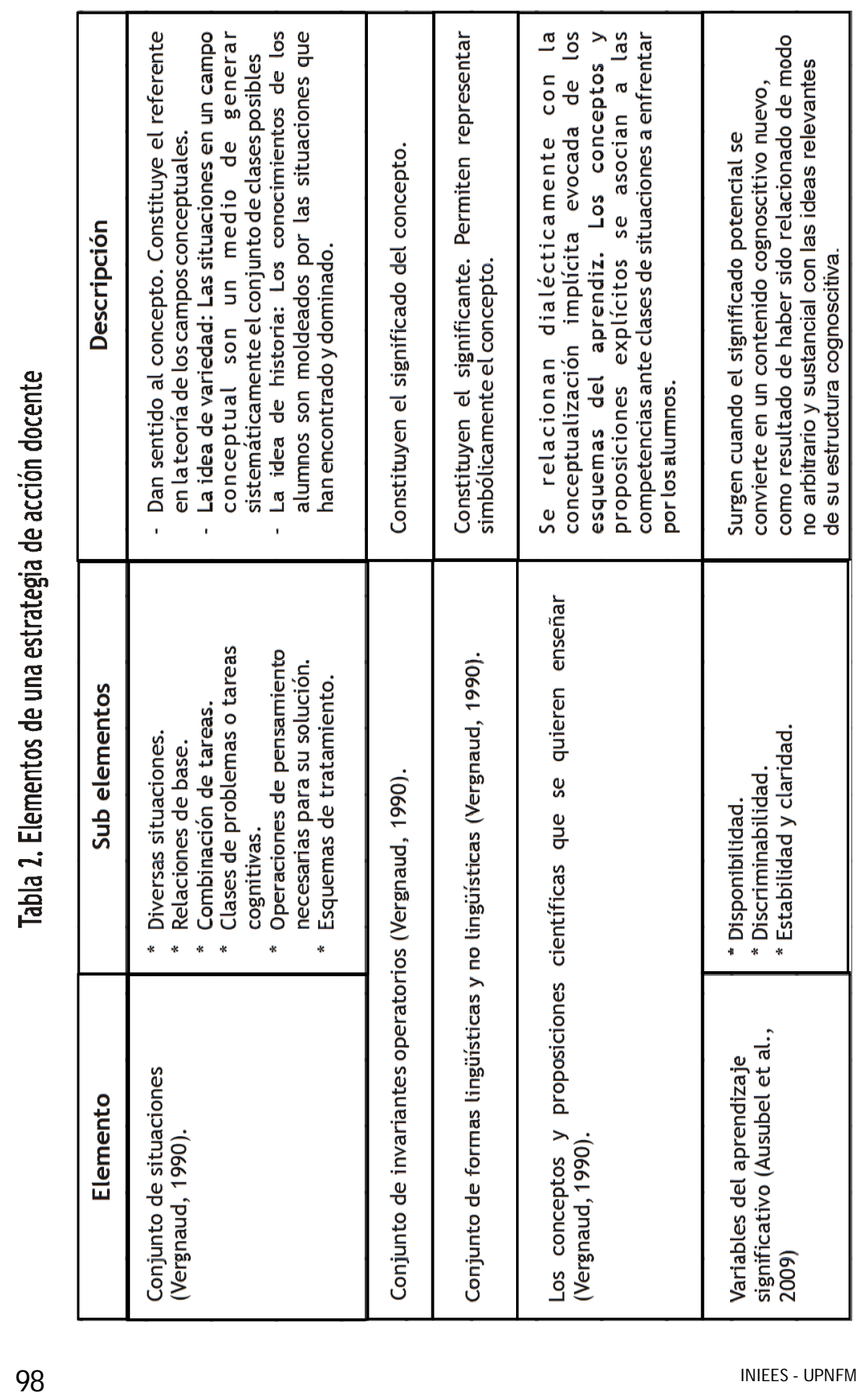


Acción docente en situaciones de aprendizaje conceptual

\begin{tabular}{|c|c|c|c|c|}
\hline 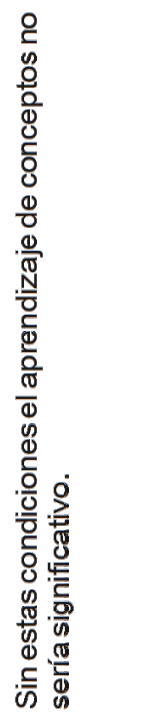 & 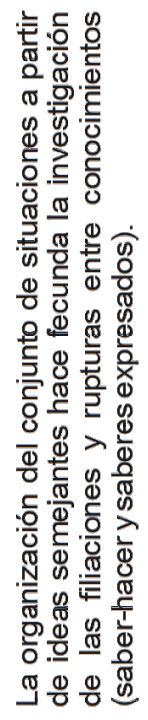 & 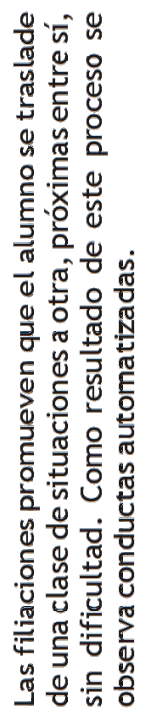 & 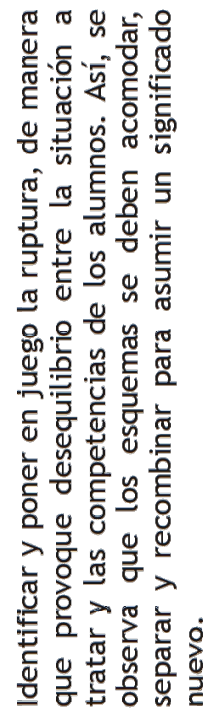 & 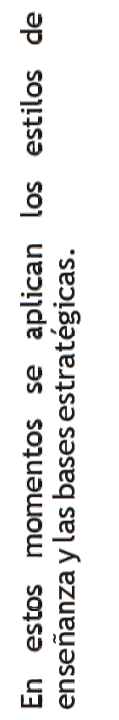 \\
\hline 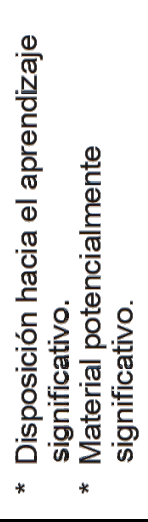 & \multirow{2}{*}{ 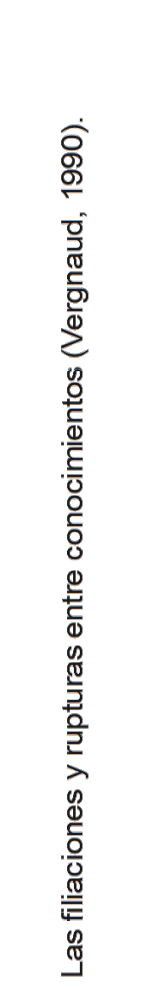 } & \multirow{2}{*}{ 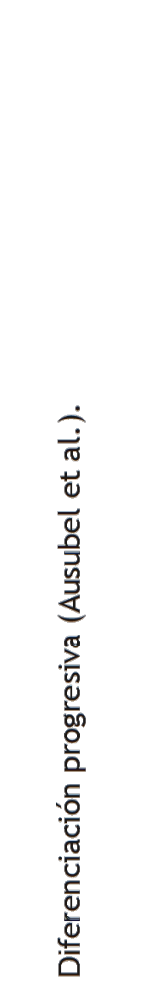 } & \multirow{2}{*}{ 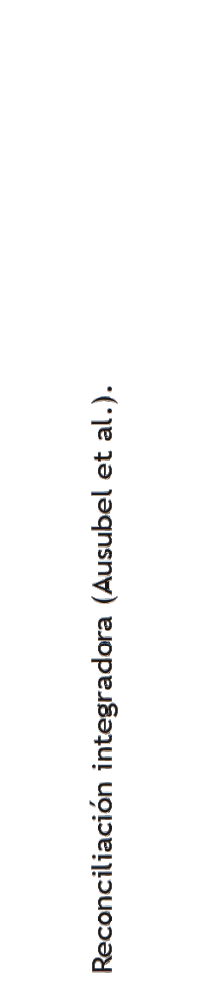 } & 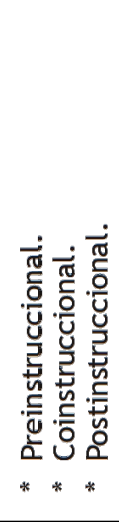 \\
\hline 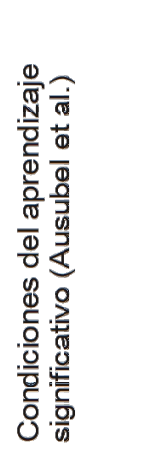 & & & & 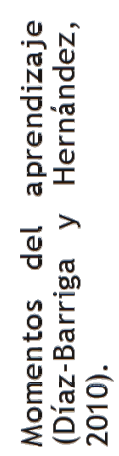 \\
\hline
\end{tabular}

<Paradigma> - Revista de Investigación Educativa. Año 21. No. 34 
Ramón Martínez, Xiomara Arrieta, Víctor Riveros

\begin{tabular}{|c|c|c|c|c|c|c|c|}
\hline 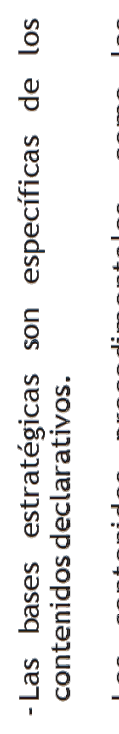 & 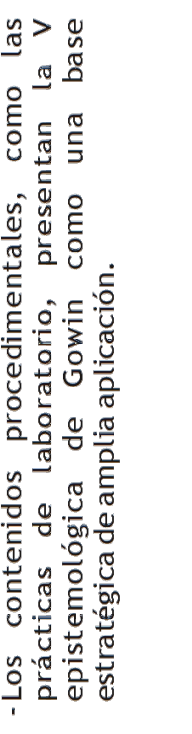 & 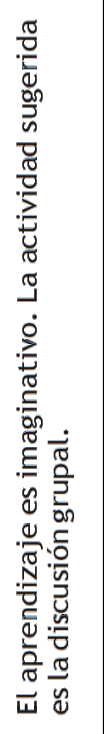 & 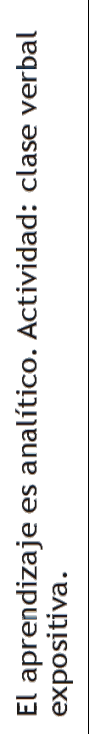 & 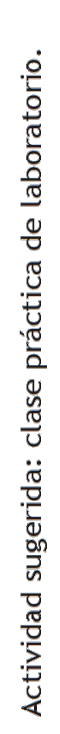 & 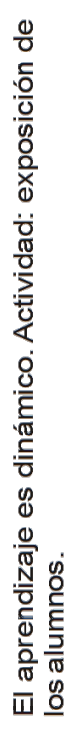 & 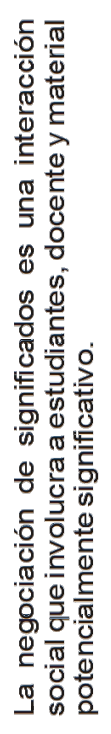 & 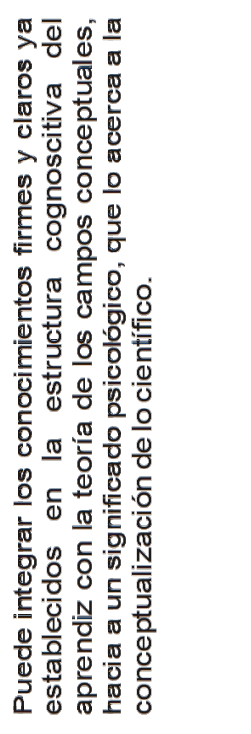 \\
\hline 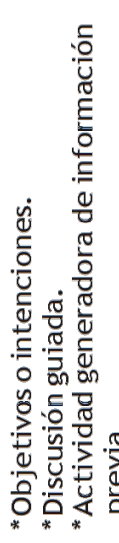 & 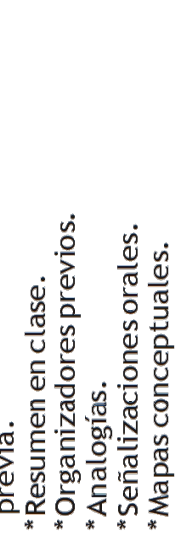 & 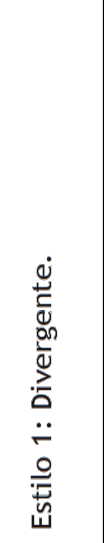 & 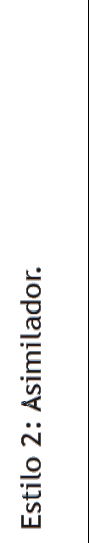 & 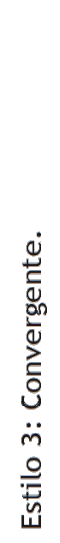 & 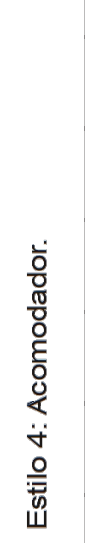 & 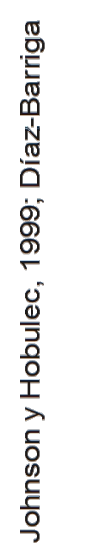 & 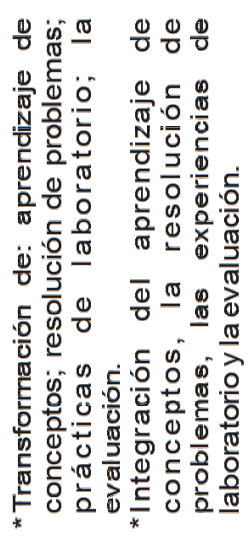 \\
\hline 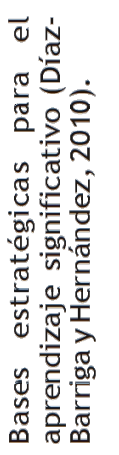 & & 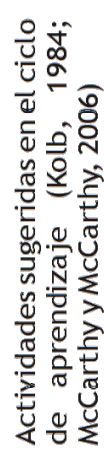 & & & & 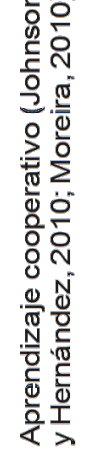 & 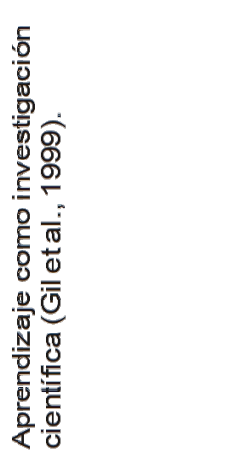 \\
\hline
\end{tabular}




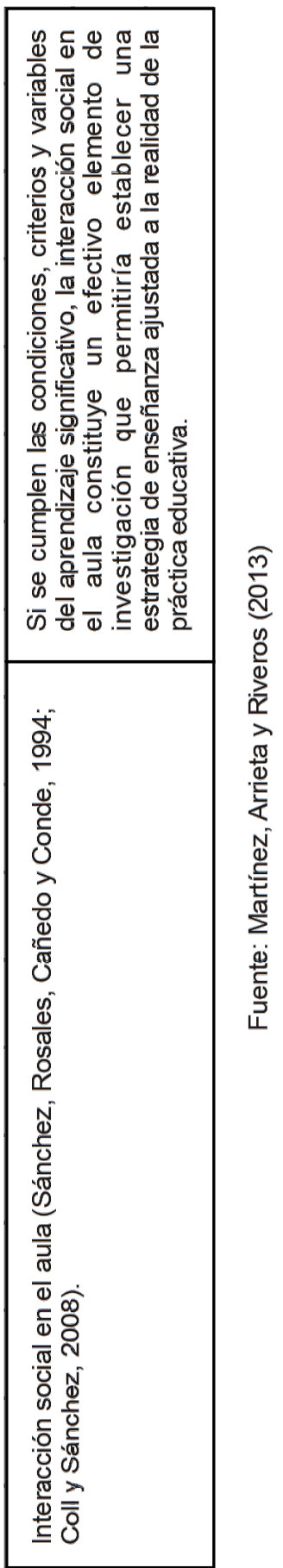

<Paradigma> - Revista de Investigación Educativa. Año 21. No. 34 


\section{La estrategia de enseñanza}

Como propuesta de la presente investigación, se diseñó una estrategia de enseñanza eficaz hacia el resultado observable del aprendizaje significativo de la conceptualización de la realidad científica, en el contexto del aula universitaria, a partir de los elementos de diseño previamente descritos.

\section{La propuesta se secuencia en fases (Ver tabla 3).}

Tabla 3. La estrategia de enseñanza

\begin{tabular}{|c|c|}
\hline Fase & Descripción \\
\hline $\begin{array}{l}\text { Se establecen los campos } \\
\text { conceptuales objeto de } \\
\text { estudio. }\end{array}$ & $\begin{array}{l}\text { La elección dependerá de los objetivos o } \\
\text { competencias de la asignatura, así como del plan } \\
\text { de estudios. }\end{array}$ \\
\hline $\begin{array}{l}\text { Se elabora el conjunto de } \\
\text { situaciones. }\end{array}$ & $\begin{array}{l}\text { El conjunto de situaciones se adapta a las } \\
\text { competencias establecidas para la asignatura. }\end{array}$ \\
\hline $\begin{array}{l}\text { Se identifican los } \\
\text { conceptos y proposiciones } \\
\text { explícitos para cada } \\
\text { situación en particular. }\end{array}$ & $\begin{array}{l}\text { Representa la sustancialidad del aprendizaje } \\
\text { significativo. }\end{array}$ \\
\hline $\begin{array}{l}\text { Se elabora las relaciones de } \\
\text { base para cada situación. }\end{array}$ & $\begin{array}{l}\text { Cada una de ellas presenta tareas y subtareas que } \\
\text { pueden estar asociados o no a la resolución de } \\
\text { problemas, sean éstos abiertos o no. }\end{array}$ \\
\hline $\begin{array}{l}\text { Se aplica el cuestionario de } \\
\text { estilos de aprendizaje de } \\
\text { Kolb y el test de hemisferios } \\
\text { cerebrales. }\end{array}$ & $\begin{array}{l}\text { Para determinar las preferencias de estilos de } \\
\text { aprendizaje individual y la inclinación de } \\
\text { hemisfericidad cerebral, respectivamente, del } \\
\text { alumnado, y así enfatizar las etapas del ciclo de } \\
\text { aprendizaje experiencial. }\end{array}$ \\
\hline $\begin{array}{l}\text { Planificación de los } \\
\text { aspectos de trabajo para el } \\
\text { aprendizaj e cooperativo } \\
\text { (Johnson et al., 1999; Díaz- } \\
\text { Barriga y Hernández, 2010) }\end{array}$ & $\begin{array}{l}\text { Dos aspectos: } \\
\text { - Decisiones previas y ejecución de tareas } \\
\text { - Trabajo en equipo } \\
\text { Una vez conformado los grupos cooperativos y } \\
\text { establecidas las tareas a desarrollar, el profesor } \\
\text { debe decidir, antes de cada situación, el } \\
\text { conjunto de situaciones o campo conceptual, } \\
\text { los criterios que empleará para evaluar el } \\
\text { desempeño de los alumnos y cómo registrará la } \\
\text { información a evaluar, en conjunción con el }\end{array}$ \\
\hline
\end{tabular}




\begin{tabular}{|c|c|}
\hline & $\begin{array}{l}\text { proceso de enseñanza y aprendizaje } \\
\text { previamente diseñado. }\end{array}$ \\
\hline $\begin{array}{l}\text { Estilo 1: Aprendizaje } \\
\text { divergente }\end{array}$ & $\begin{array}{l}\text { - Se identifican, estiman y estudian los } \\
\text { conocimientos previos. Se realizan la evaluación } \\
\text { diagnóstica o predictiva y las bases estratégicas } \\
\text { como los organizadores previos y los mapas } \\
\text { conceptuales. Esto permite la programación de } \\
\text { los contenidos del campo conceptual y ayuda a } \\
\text { los estudiantes a tomar conciencia sobre la } \\
\text { experiencia próxima de aprendizaje, generar } \\
\text { expectativas apropiadas sobre lo que va } \\
\text { aprender, activar sus invariantes operatorios y } \\
\text { motivarlos. Los alumnos comienzan a aprender } \\
\text { conceptos desde el momento en que se les } \\
\text { evalúa inicialmente. }\end{array}$ \\
\hline $\begin{array}{l}\text { Estilo 2: Aprendizaje } \\
\text { asimilador }\end{array}$ & $\begin{array}{l}\text { - La estrategia de enseñanza es la clase } \\
\text { tradicional. Se asocia al aprendizaje de } \\
\text { conceptos y a la resolución de problemas. } \\
\text { - La base estratégica recomendadas son los mapas } \\
\text { conceptuales (Moreira, 2010). } \\
\text { - La evaluación constructivista de esta fase se } \\
\text { divide en dos tipos: formativa y sumativa (Díaz- } \\
\text { Barriga y Hernández, 2010; J ohnson et al.). }\end{array}$ \\
\hline $\begin{array}{l}\text { Estilo 3: Aprendizaje } \\
\text { convergente }\end{array}$ & $\begin{array}{l}\text { - Se asocia a la etapa de las prácticas de } \\
\text { laboratorio. La base estratégica más utilizada } \\
\text { es la V epistemológica de Gowin. } \\
\text { - Se fortalece con los aportes de la teoría de los } \\
\text { campos conceptuales de Vergnaud (1990, 2007) } \\
\text { que conllevan a un modelo dinámico de } \\
\text { aprendizaje en los trabajos de laboratorio en } \\
\text { física (Andrés et al., 2006). }\end{array}$ \\
\hline $\begin{array}{l}\text { Estilo 4: Aprendizaje } \\
\text { acomodador }\end{array}$ & $\begin{array}{l}\text { Se representa y exponen los mapas conceptuales } \\
\text { como función social acreditativa de la evaluación } \\
\text { sumativa. Se evalúa el resultado observable de la } \\
\text { conceptualización científica alcanzada por el } \\
\text { alumno. }\end{array}$ \\
\hline $\begin{array}{l}\text { Se diagnostica la calidad y } \\
\text { cantidad del aprendizaje } \\
\text { como evaluación sumativa } \\
\text { final. }\end{array}$ & $\begin{array}{l}\text { Los resultados observables del aprendizaje } \\
\text { significativo de la conceptualización científica de } \\
\text { competencias u objetivos, particulares o ge- } \\
\text { nerales, son analizados y publicados por el } \\
\text { docente, y discutido con sus estudiantes. }\end{array}$ \\
\hline
\end{tabular}

Fuente: Martínez, Arrieta y Riveros (2013) 
Ramón Martínez, Xiomara Arrieta, Víctor Riveros

La figura 2 representa las interconexiones de las integraciones descritas, los elementos y fases de la estrategia de enseñanza.

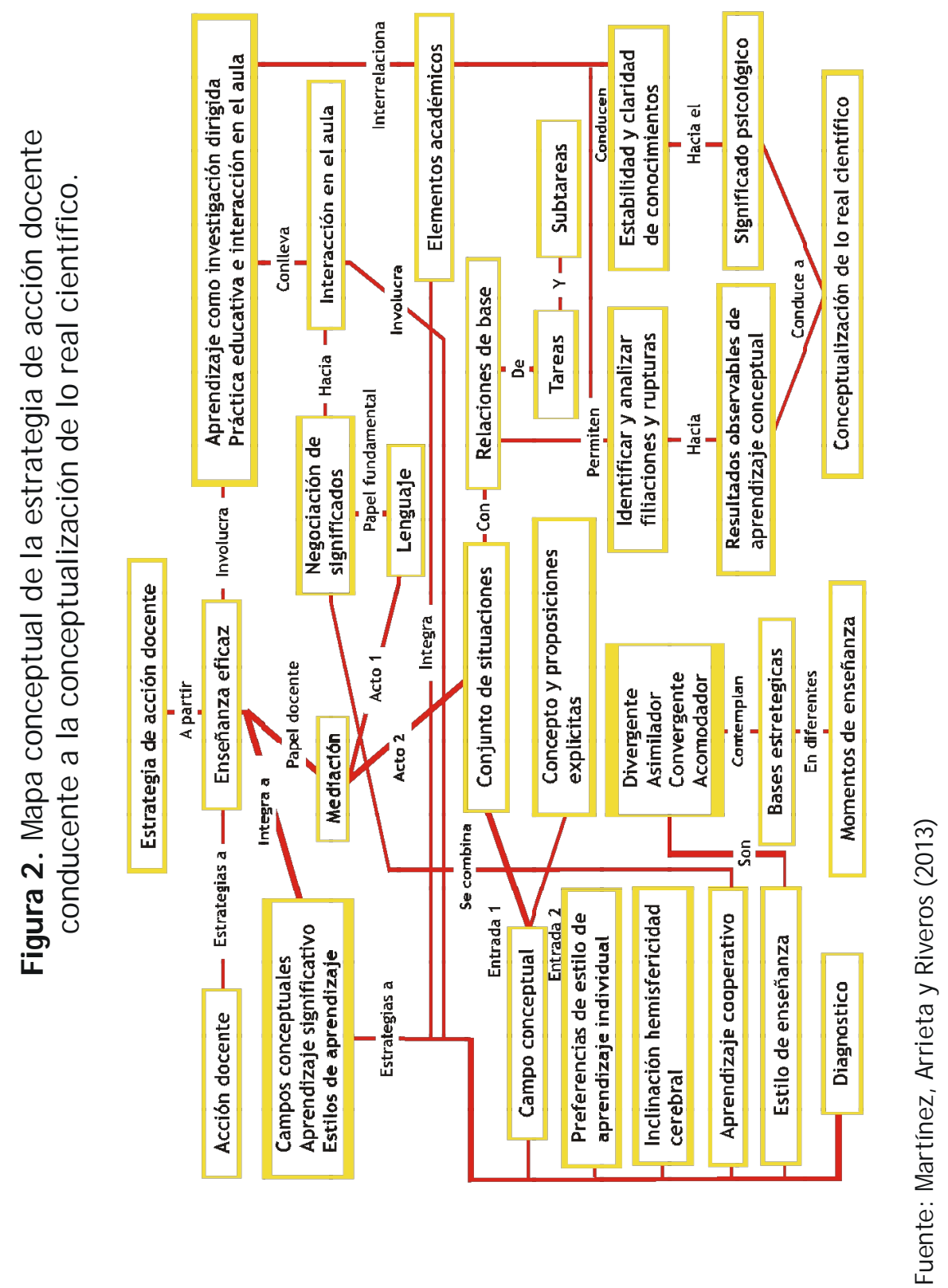




\section{Consideraciones finales}

La estrategia de enseñanza, se basa en el concepto de enseñanza eficaz, específica para este estudio. La enseñanza eficaz presenta dos condiciones: una necesaria, que involucra la integración de los constructos campos conceptuales, aprendizaje significativo y estilos de aprendizaje individual, y una suficiente, que involucra el aprendizaje como investigación dirigida y el estudio de la práctica educativa, específicamente la interacción en el aula. Ambas condiciones, a la postre, conducen a la conceptualización de lo real científico.

Las fases de la estrategia de enseñanza integran los elementos académicos hacia un aprendizaj e como investigación científica, como parte del aprendizaj e significativo de la conceptualización científica, y están condicionadas por la disposición del aprendiz por aprender significativamente y la utilización de un material de instrucción potencialmente significativo, que posea significado lógico y ancle con las ideas de afianzamiento pertinentes y relevantes en la estructura cognoscitiva del aprendiz. Para alcanzar una integración efectiva es necesario que estos elementos académicos se transformen de la manera tradicional a una reorientación enfocada en la forma en que el científico enfrenta la problemática científica real.

El significado psicológico conducente a la conceptualización científica combina el aprendizaje como investigación científica con la estructura cognoscitiva más estable y clara obtenida por el alumno en el proceso de enseñanza y aprendizaje significativo. Ambas se relacionan a su vez con el acoplamiento teórico esquema/ situación vergnaudiano, que constituye la piedra angular de la didáctica (Vergnaud, 2007).

El estudio de la interacción social en el aula involucra todas las fases de diseño establecidas con la finalidad de analizar las filiaciones y rupturas entre conocimientos de los alumnos, de manera constructivista, hacia resultados observables de aprendizaje de conceptos y proposiciones explícitos, por lo que se espera que al aplicar la estrategia se promueva una enseñanza eficaz y por consiguiente un aprendizaje significativo de la conceptualización de 
la realidad científica por parte de los estudiantes.

La conceptualización de lo real científico es el resultado del significado psicológico, una vez alcanzada la estabilidad y claridad de los conocimientos adquiridos por los alumnos, así como los resultados observables del aprendizaje significativo, producto del análisis de las filiaciones y rupturas entre los conocimientos implícitos y explícitos. La estabilidad y claridad y los resultados observables del aprendizaj e significativo son el resultado de las fases de la estrategia, la integración de los elementos académicos y el estudio de la interacción en el aula. Las fases de la estrategia, a su vez, son el resultado de la integración de los constructos nombrados en el parágrafo anterior y se construyen a partir de las preferencias de los estilos de aprendizaje individual, que conducen a los estilos de enseñanza: divergente, asimilador, convergente y acomodador.

La acción docente proporciona las estrategias de tipo cognoscitivo, de apoyo y facilitadoras a la enseñanza eficaz. El papel principal del docente en la actividad de negociación de significados es el de mediador. El papel del lenguaje es fundamental en este proceso. El conjunto de situaciones constituyen el referente de la conceptualización de lo real científico. Ambos son actos de este papel mediador. La negociación de significados es un proceso que involucra el aprendizaj e cooperativo como una forma de interacción en el aula de clase.

Por todo lo anterior, la estrategia planteada constituye una forma de aprendizaj e centrada en el alumno. 
Acción docente en situaciones de aprendizaje conceptual

\section{Referencias bibliográficas}

Andrés, M.; Pesa, M.; Moreira, M. (2006). El trabajo de laboratorio en cursos de física desde la teoría de los campos conceptuales. Ciência \& Educação (Bauru), Vol. 12, № 2, pp.129-142.

Ausubel, D.; Novak, J .; Hanesian, H. (2009). Psicología educativa. Un punto de vista cognoscitivo. Segunda edición reimpresa (en español), traducción: Mario Sandoval Pineda, México: Editorial Trillas, p. 623.

Coll, C.; Sánchez, E. (2008). El análisis de la interacción alumnoprofesor: líneas de investigación. Revista de educación, $N^{\circ} 346$, pp. 15-32.

Cubero, Rosario; Cubero, Mercedes; Santamaría, Andrés; De la Mata, Manuel; Ignacio, María; Prados, María (2008). La educación a través de su discurso. Prácticas educativas y construcción discursiva del conocimiento en el aula. Revista de Educación; $N^{\circ} 346$, pp. 71104.

Díaz-Barriga, F.; Hernández, G. (2010). Estrategias docentes para un aprendizaje significativo: una interpretación constructivista. Segunda edición, México: McGraw-Hill, p. 405.

Gil, D.; Furió, C.; Valdez, P.; Salinas, J.; Martínez-Torregosa, J.; Guisasola, J., González, E.; Dumas-Varré, A.; Goffard, M.; Pessoa, A. (1999). ¿Tiene sentido seguir distinguiendo entre aprendizaje de conceptos, resolución de problemas de lápiz y papel y realización de prácticas de laboratorio? Enseñanza de las ciencias, Vol. 17, $\mathrm{N}^{\circ} 2$, pp. 311-320.

Gowin, D. (1981). Educating. 1aedición, EEUU: Cornell University Press, p. 210.

Keefe, J. y Ferrell, B. (1990). Developing a DefensibleLearning Style Paradigm [Documento en línea]. Disponible: http:/ / 12.4.125.3/ ASCD/pdf/journals/ ed_lead/ el_199010_keefe.pdf [Consulta: 2012, Marzo 9]. 
Kolb, D. (1984). Experiential learning: experience as the Source of Learning and Development, $1^{\text {a }}$ edición, EEUU: Prentice-Hall, $p$. 256.

Johnson, D.; Johnson, R.; Holubec, E. (1999). El aprendizaje cooperativo en el aula. Primera edición, Buenos Aires, Argentina: Editorial Paidós, p. 66.

McCarthy, B.; McCarthy, D. (2006). Teaching Around the 4MAT Cycle. Primera edición, California, EEUU: Corwin Press A Sage Publications Company, p. 99.

Meleán, R.; Arrieta, X. (2009). Estrategia didáctica para el desarrollo de esquemas en resolución de problemas según la teoría de los campos conceptuales. Sapiens, Año 10, N², pp. 69-96.

Moreira, M. (2010). ¿Por qué conceptos? ¿Por qué actividades colaborativas? ¿Por qué mapas conceptuales? Qurriculum, $\mathrm{N}^{\circ} 23$, pp. 9-23.

Salas Silva, R. (2008). Estilos de aprendizaje a la luz de la neurociencia. Primera edición, Bogotá, Colombia: Cooperativa Editorial Magisterio, p. 412.

Sánchez, E.; Rosales, J.; Cañedo, I.; Conde, P. (1994). El discurso expositivo: una comparación entre profesores expertos y principiantes. Infancia y Aprendizaje, pp. 51-74.

Vergnaud, G. (1990). La Teoría de Ios Campos Conceptuales. Recherches en Didáctique des Mathématiques [Revista en línea], 10. Disponible: http:// ipes. anep. edu.uy/ documentos/curso_dir_07/ modulo2/ materiales/ didactica/campos. pdf[Consulta: $2 \overline{0} 12$, Noviembre 20].

Vergnaud G. (2007). ¿En qué sentido la Teoría de los Campos Conceptuales puede ayudarnos para facilitar aprendizaje significativo? Investigaciones em Ensino de Ciencias. V12 (2). pp. 285-302 


\title{
Nota sobre los autores
}

\begin{abstract}
Ramón Martínez
Ingeniero Electricista, egresado de la Universidad de Oriente (UDO), Venezuela, en 1992. Magister Scientiarum en Educación, Mención Planificación Educativa, egresado de la UDO, en 2004. Doctorando en Ciencias Humanas de La Universidad del Zulia (LUZ), Venezuela. Profesor Agregado a Dedicación Exclusiva en el Núcleo de Bolívar de la UDO, en el Área de Física. Miembro activo del Grupo de Investigación Cualitativo en el mencionado Núcleo. Miembro activo de la Asociación Venezolana para el Avance de la Ciencia (AsoVAC), Capítulo Guayana. ramonfisicaudo@gmail.com
\end{abstract}

\section{Xiomara Arrieta}

Profesora Titular a Dedicación Exclusiva de la Universidad del Zulia, Facultad de Humanidades y Educación, Departamento de Matemática y Física. Técnico Químico, Licenciada en Educación, mención Cs. Matemáticas. Magíster Scientiarum en Matemática Aplicada, Facultad de Ingeniería. Magíster Scientiarum en Ciencias Aplicadas, área Física, Facultad de Ingeniería. Doctora en Ciencias Humanas, Postdoctorado en Ciencias Humanas, Facultad de Humanidades y Educación. Autora y coautora de artículos publicados en revistas nacionales e internacionales. Investigadora activa del Centro de Estudios Matemáticos y Físicos y de la línea Didáctica de la Matemática y las Ciencias Naturales del Doctorado en Ciencias Humanas, con proyectos inscritos en el Consejo de Desarrollo Científico y Humanístico (CONDES). Investigadora acreditada al PEII, nivel C. xarrieta2410@yahoo.com 
Ramón Martínez, Xiomara Arrieta, Víctor Riveros

\section{Víctor Riveros}

Profesor Titular a Dedicación Exclusiva de la Universidad del Zulia, Facultad de Humanidades y Educación, Departamento de Matemática y Física. Licenciado en Educación, mención Cs. Matemáticas. Magíster Scientiarum en Matemática Aplicada, Facultad de Ingeniería. Doctor en Ciencias Humanas, Post doctorado en Ciencias Humanas, Facultad de Humanidades y Educación. Autor y coautor de artículos publicados en revistas nacionales e internacionales. Investigador activo del Centro de Estudios Matemáticos y Físicos y de la línea Didáctica de la Matemática y las Ciencias Naturales del Doctorado en Ciencias Humanas, con proyectos inscritos en el Consejo de Desarrollo Científico y Humanístico (CONDES). Investigador acreditado al PEll, nivel B. vriveros75@gmail.com 